\title{
Weighted Pseudo Almost Automorphic Solutions to Non- autonomous Semilinear Differential Equations
}

\author{
Saud M. Alsulami \\ Mathematics Department, King Abdulaziz University \\ P.O.Box: 138381 Jeddah 21323 Saudi Arabia \\ E-mail: alsulami@kau.edu.sa
}

Received February 23, 2010 / Accepted May 28, 2010

\begin{abstract}
We consider the existence and uniqueness of Weighted Pseudo almost automorphic solutions to the non-autonomous semilinear differential equation in a Banach space $X$ :

$$
u^{\prime}(t)=A(t) u(t)+f(t, u(t)), t \in \mathrm{R}
$$

where $A(t), t \in \mathrm{R}$, generates an exponentially stable evolution family $\{U(t, s)\}$ and $f: \mathrm{R} \times X \rightarrow X$ satisfies a Lipschitz condition with respect to the second argument.

MSC 2010: 43A60; 34G20, 47Dxx
\end{abstract}

Keywords: Weighted Pseudo Almost automorphic; Non-autonomous equations; Semilinear differential equations.

\section{Introduction}

Bochner introduced the concept of almost automorphic functions in his papers ([3]-[5]) in relation to some aspects of differential geometry. This concept became a generalization of almost periodicity which is one of the most attractive topics in the qualitative theory of differential equations because of its significance and applications in physics, mathematical biology, control theory, and other related fields. A natural generalization of the concept of almost automorphic functions is the concept of pseudo almost automorphic functions which has widely been used in investigation of the existence of almost automorphic solutions of various kinds of evolution equations by many others. For more information of the latter concept, we refer the reader to [2], [7] and [9]. In [6], a new class of functions called weighted pseudo almost automorphic functions was introduced, which generalize in a natural fashion weighted pseudo almost periodic functions due to T. Diagana, and moreover, pseudo almost automorphic functions due to J. Liang, J. Zhang and T.J. Xiao. 
In this paper we study the existence and uniqueness of weighted pseudo almost automorphic solutions to the following non-autonomous semilinear differential equation in a Banach space $X$ :

$$
u^{\prime}(t)=A(t) u(t)+f(t, u(t)), t \in \mathrm{R}
$$

where $U(t, s)$ generated by $A(t)$, is exponentially stable and $f: \mathrm{R} \times X \rightarrow X$ is a weighted pseudo almost automorphic function which satisfies a Lipschitz condition with respect to the second argument.

\section{Preliminaries}

Definition 2.1 (i) A continuous function $f: \mathrm{R} \rightarrow X$ is said to be almost automorphic (in Bochner's sense) if for every sequence of real numbers $\left\{s_{n}^{\prime}\right\}$, there exists a subsequence $\left\{s_{n}\right\}$ such that

$$
g(t):=\lim _{n \rightarrow \infty} f\left(t+s_{n}\right)
$$

is well defined for each $t \in \mathrm{R}$, and

$$
\lim _{n \rightarrow \infty} g\left(t-s_{n}\right)=f(t)
$$

for each $t \in \mathrm{R} . A A(\mathrm{R}, X)$ stands for the set of all such functions.

(ii) A continuous function $f: \mathrm{R} \times X \rightarrow X$ is said to be almost automorphic if $f(t, x)$ is almost automorphic in $t \in \mathrm{R}$ uniformly for all $x$ in any bounded subsets of $X . A A(\mathrm{R} \times X, X)$ is the set of all such functions.

(iii) A continuous function $f: \mathrm{R} \rightarrow X$ (resp. $f: \mathrm{R} \times X \rightarrow X$ ) is said to be pseudo almost automorphic if it can be decomposed as $f=g+\phi$ where $g \in A A(\mathrm{R}, X)(\operatorname{resp} . A A(\mathrm{R} \times X, X)$ ) and $\phi$ is bounded continuous function with vanishing mean value (resp. $\phi$ is bounded continuous function with

$$
\lim _{T \rightarrow \infty} \frac{1}{2 T} \int_{-T}^{T} \mathrm{P} \phi(\sigma, x) \mathrm{P} d \sigma=0
$$

uniformly for all $x$ in any bounded subsets of $X$ ). Denote by $P A A(\mathrm{R}, X)$ (resp. $P A A(\mathrm{R} \times X, X))$ the set of all such functions.

Note that $\left(P A A(\mathrm{R}, X), \mathrm{P} \cdot \mathrm{P}_{0}\right)$ turns out to be a Banach space, where $\mathrm{P} \cdot \mathrm{P}_{0}$ is the supremum norm, see [10, Theorem 2.2].

Throughout this paper, $X$ stands for a Banach space with norm P.P. We denote by $C(\mathrm{R}, X)$ the Banach space of all continuous functions from $\mathrm{R}$ to $X$. Similarly, $B C(\mathrm{R}, X)$ is the Banach space of all bounded continuous functions from $\mathrm{R}$ to $X$. Note that $\left(B C(\mathrm{R}, X), \mathrm{P} \cdot \mathrm{P}_{\infty}\right)$ is a Banach space with the sup norm P.P.

Note that $\left(A A(X), \mathrm{P} \cdot \mathrm{P}_{\infty}\right)$ turns out to be a Banach space.

Now like in [8], let $U$ denote the collection of functions (weights) $\rho: \mathrm{R} \rightarrow(0, \infty)$, which are locally integrable over $\mathrm{R}$ such that $\rho>0$ almost everywhere.

$$
\text { For a given } r>0 \text {, set } \mu(r, \rho):=\int_{-r}^{r} \rho(x) d x \text { for each } \rho \in U \text {. }
$$


Define $U_{\infty}:=\left\{\rho \in U: \lim _{r \rightarrow \infty} \mu(r, \rho)=\infty\right\}$

and $U_{b}:=\left\{\rho \in U_{\infty}: \rho\right.$ is bounded and $\left.\inf _{x \in \mathrm{R}} \rho(x)>0\right\}$.

Note that $U_{b} \subset U_{\infty} \subset U$.

For $\rho \in U_{\infty}$, define

$P A A_{0}(\mathrm{R}, \rho):=\left\{f \in B C(\mathrm{R}, X): \lim _{r \rightarrow \infty} \frac{1}{\mu(r, \rho)} \int_{-r}^{r}\|f(s)\| \rho(s) d s=0\right\}$.

In a similar manner, we define $P A A_{0}(\mathrm{R} \times X, \rho)$.

The sets $W P A A(\mathrm{R}, \rho)$ and $W P A A(\mathrm{R} \times X, \rho)$ of weighted pseudo almost automorphic functions are defined as:

$$
\begin{gathered}
W P A A(\mathrm{R}, \rho):=\left\{\begin{array}{c}
f=g+\phi \in B C(\mathrm{R}, X): \\
g \in A A(\mathrm{R}, X) \operatorname{and} \phi \in P A A_{0}(\mathrm{R}, \rho)
\end{array}\right\} ; \\
W P A A(\mathrm{R} \times X, \rho):=\left\{\begin{array}{c}
f=g+\phi \in B C(\mathrm{R} \times X, X): \\
g \in A A(\mathrm{R} \times X, X) \operatorname{and} \phi \in P A A_{0}(\mathrm{R} \times X, \rho)
\end{array}\right\} .
\end{gathered}
$$

We recall the following results:

Theorem 2.2 [6] : The decomposition of a weighted pseudo almost automorphic function is unique for any $\rho \in U_{b}$.

Theorem 2.3 [6] : If $\rho \in U_{b}$, then (WPAA( $\left.\left.\mathrm{R}, \rho\right),\|\cdot\|_{\infty}\right)$ is a Banach space.

We make the following assumptions

H1. $f(t, x)$ is uniformly continuous in any bounded subset $K \subset X$ uniformly in $t \in \mathrm{R}$.

H2. $g(t, x)$ is uniformly continuous in any bounded subset $K \subset X$ uniformly in $t \in \mathrm{R}$.

Let us recall Theorem 2.4 from [9] :

Theorem $2.4 \quad$ Let $\quad f=g+\phi \in P A A(\mathrm{R} \times X, X) \quad$ where $g(t, u) \in A A(\mathrm{R} \times X, X), \phi(t, u) \in A A_{0}(\mathrm{R} \times X, X)$ such that $\mathrm{H} 1$ and $\mathrm{H} 2$ are satisfied. If $u(t) \in P A A(\mathrm{R}, X)$, then $f(\cdot, u(\cdot)) \in P A A(\mathrm{R}, X)$.

Moreover, we have [6, Theorem 2.10] :

Theorem 2.5 Let $f=g+\phi \in W P A A(\mathrm{R}, \rho)$ where $\rho \in U_{\infty}$ and assume that $H 1$ and $H 2$ are satisfied. If $u \in W P A A(\mathrm{R}, \rho)$, then $f(\cdot, u(\cdot)) \in W P A A(\mathrm{R}, \rho)$.

Corollary 2.6 Let $f=g+\phi \in W P A A(\mathrm{R}, \rho)$ where $\rho \in U_{\infty}$ and assume both $f$ and $g$ are Lipschitzian in $x \in X$ uniformly in $t \in \mathrm{R}$. If $u \in W P A A(\mathrm{R}, \rho)$, then $f(\cdot, u(\cdot)) \in W P A A(\mathrm{R}, \rho)$.

In this paper we assume that $\{A(t)\}_{t \in \mathrm{R}}$ satisfies the 'Acquistapace-Terreni' conditions introduced in ([1]), that is, 
H3. there exist constants $\lambda_{0} \geq 0, \theta \in\left(\frac{\pi}{2}, \pi\right), L, K \geq 0$, and $\alpha, \beta \in(0,1]$ with $\alpha+\beta>1$ such that

and

$$
\Sigma_{\theta} \cup\{0\} \subset \rho\left(A(t)-\lambda_{0}\right), \mathrm{P} R\left(\lambda, A(t)-\lambda_{0}\right) \mathrm{P} \leq \frac{K}{1+|\lambda|}
$$

$$
\mathrm{P}\left(A(t)-\lambda_{0}\right) R\left(\lambda, A(t)-\lambda_{0}\right)\left[R\left(\lambda_{0}, A(t)\right)-R\left(\lambda_{0}, A(s)\right)\right] \mathrm{P} \leq L|t-s|^{\alpha}|\lambda|^{-\beta}
$$

for $t, s \in \mathrm{R}, \lambda \in \Sigma_{\theta}:=\{\lambda \in \mathrm{C} \backslash\{0\}:|\arg \lambda| \leq \theta\}$.

Remark : If the condition (H3) holds, then there exists a unique evolution family $\{U(t, s)\}_{-\infty<s \leq t<\infty}$ on $X$, which satisfies the homogenous equation $u^{\prime}(t)=A(t) u(t), t \in \mathrm{R}$.

\section{Main Results}

Consider

$$
u^{\prime}(t)=A(t) u(t)+f(t, u(t)), t \in \mathrm{R}
$$

Definition 3.1 A mild solution to (1) is a continuous function $u(t): \mathrm{R} \rightarrow X$ satisfying

$$
u(t)=U(t, a) u(a)+\int_{a}^{t} U(t, s) f(s, u(s)) d s
$$

for all $t \geq a$ and all $a \in \mathrm{R}$.

In the proof of the following theorem we follow the same reasoning as in the proof of Theorem 3.3 in [10] with the proper modification .

Theorem 3.2 Suppose that the evolution family $U(t, s)$ generated by $A(t)$ is exponentially stable, that is, there are constants $K, \omega>0$ such that $\mathrm{PU}(t, s) \mathrm{P} \leq K e^{-\omega(t-s)}$ for all $t \geq s$. and $f=g+\phi \in W P A A(\mathrm{R} \times X, \rho), \rho \in U_{b}$, satisfies

$$
\begin{aligned}
& \mathrm{P} f(t, u)-f(t, v) \mathrm{P} \leq L_{f} \mathrm{P} u-v \mathrm{P} \\
& \mathrm{P} g(t, u)-g(t, v) \mathrm{P} \leq L_{g} \mathrm{P} u-v \mathrm{P}
\end{aligned}
$$

$t \in \mathrm{R}$ and $u, v \in X$ for $L<\frac{\omega}{K}$. Then the equation (1) has a unique weighted pseudo almost automorphic mild solution.

Proof.

Consider the nonlinear operator $\mathrm{F}$ given by

$$
\left(\mathrm{F} u \psi(t)=\int_{-\infty}^{t} U(t, s) f(s, u(s)) d s\right.
$$


If $u(s) \in W P A A(\mathrm{R}, X)$, then corollary 2.11 in [6] gives that $f(s, u(s)) \in W P A A(\mathrm{R}, X)$. i.e., there exist $g \in A A(\mathrm{R}, X)$ and a bounded continuous function $\phi$ with vanishing mean value, such that $f=g+\phi$. Therefore $(\mathrm{F} u)(t)$ can be expressed as $(\mathrm{F} u)(t)=G(t)+\Phi(t)$, where

$$
\begin{aligned}
& G(t)=\int_{-\infty}^{t} U(t, s) g(s, u(s)) d s . \\
& \Phi(t)=\int_{-\infty}^{t} U(t, s) \phi(s, u(s)) d s .
\end{aligned}
$$

From the proof of N'Guerekata [[1 1], Theorem 3.2], it follows that $t \rightarrow G(t)$ is almost automorphic.

Next, we show that $\Phi(t)$ is a bounded continuous function with vanishing mean value. Since $\Phi(t)$ is bounded on $\mathrm{R}$, we have $M:=\sup _{t \in \mathrm{R}} \mathrm{P} \Phi(t) \mathrm{P}<+\infty$. For any $T \in[-t, t]$, we get

$$
\int_{-T}^{T} \int_{-\infty}^{-T} \mathrm{P} U(t, s) \Phi(s) \mathrm{P} d s d t \leq \frac{M K}{\omega^{2}}
$$

On the other hand,

$$
\int_{-T}^{T} \int_{-T}^{t} \mathrm{P} U(t, s) \Phi(s) \mathrm{P} d s d t \leq \frac{K}{\omega} \int_{-T}^{T} \mathrm{P} \Phi(s) \mathrm{P} d s
$$

By the above two equations, we get

$$
\lim _{T \rightarrow \infty} \int_{-T}^{T} \int_{-\infty}^{t} \mathrm{P} U(t, s) \Phi(s) \mathrm{P} d s d t=0
$$

Thus, $\Phi(t)$ is a bounded continuous function with vanishing mean value. Therefore, $(\mathrm{F} u)(t)=G(t)+\Phi(t)$ is a weighted pseudo almost automorphic function on $\mathrm{R}$. That is, $\mathrm{F}(W P A A(\mathrm{R}, \rho)) \subseteq W P A A(\mathrm{R}, \rho)$. Moreover, for every $u, v \in W P A A(\mathrm{R}, \rho)$,

$$
\begin{gathered}
\left.\mathrm{PF} u-\mathrm{F} v \mathrm{P} \leq \sup _{t \in \mathrm{R}} \int_{-\infty}^{t} \mathrm{P} U(t, s) \mathrm{P} f(s, u(s))-f(s, v(s))\right] d s \\
\leq \frac{L K}{\omega} \mathrm{P} u-v \mathrm{P}
\end{gathered}
$$

Thus $\mathrm{F}$ is a contraction on $W P A A(\mathrm{R}, \rho)$. Therefore, by the contraction mapping theorem $\mathrm{F}$ has a unique fixed point $u(t) \in W P A A(\mathrm{R}, \rho)$ since $W P A A(\mathrm{R}, \rho)$ is complete. The fixed point satisfies the integral equation

$$
u(t)=\int_{-\infty}^{t} U(t, s) f(s, u(s)) d s,
$$

for all $t \in \mathrm{R}$. Fixing $a \in \mathrm{R}$, we have

$$
u(a)=\int_{-\infty}^{a} U(a, s) f(s, u(s)) d s,
$$

Since $U(t, s)=U(t, a) U(a, s)$, for $-\infty<s \leq a \leq t<\infty$, it follows that $u(t)$ satisfies (2). Hence $u(t)$ is a mild solution to (1).

On the other hand, let $v(t)$ be a weighted pseudo almost automorphic mild solution to (1). Then $v(t)$ satisfies the equation (2), with $u$ replaced by $v$. Letting $a \rightarrow-\infty$ yields 


$$
v(t)=\int_{-\infty}^{t} U(t, s) f(s, v(s)) d s, t \in \mathrm{R}
$$

Since $v(t)$ is bounded on $\mathrm{R}$ and $U(t, s)$ is exponentially stable. Hence $u(t) \equiv v(t)$ on $\mathrm{R}$. i.e., $u(t)$ is the unique mild solution to $(1)$.

Corollary 3.3 Suppose that the $C_{0}$ - semigroup $T(t)$ generated by $A$ is exponentially stable, that is, there are constants $K, \omega>0$ such that $\mathrm{P} T(t) \mathrm{P} \leq K e^{-\omega t}$ for all $t \geq 0$. and $f=g+\phi \in W P A A(\mathrm{R} \times X, \rho), \rho \in U_{b}$, satisfies

$$
\begin{aligned}
& \mathrm{P} f(t, u)-f(t, v) \mathrm{P} \leq L_{f} \mathrm{P} u-v \mathrm{P} \\
& \mathrm{P} g(t, u)-g(t, v) \mathrm{P} \leq L_{g} \mathrm{P} u-v \mathrm{P}
\end{aligned}
$$

$t \in \mathrm{R}$ and $u, v \in X$ for $L<\frac{\omega}{K}$. Then the equation

$$
u^{\prime}(t)=A u(t)+f(t, u(t)), t \in \mathrm{R}
$$

has a unique weighted pseudo almost automorphic mild solution.

\section{Acknowledgments}

This work has been done under the Project No. 429/40-3. The author is grateful to the Deanship of Scientific Research at King Abdulaziz University for their financial support. The author also is grateful to the referees for the valuable comments. Moreover, the author would like to thank Prof. N'Guérékata for his valuable comments.

\section{References}

[1] Acquistapace P and B Terreni (1987) A unified approach to abstract linear parabolic equations. Rend. Sem. Mat. Univ. Padova 78: 47-107.

[2] Alsulami S (2010) Pseudo Almost Automorphic Solutions of non-autonomous Semilinear Differential Equations in Banach spaces. Int. Journal of Math. Analysis 4(9): 413 - 418.

[3] Bochner S (1961) Uniform convergence of monotone sequences of functions. Proc. Natl. Acad. Sci. USA 47: 582 585.

[4] Bochner S (1962) A new approach to Almost periodicity. Proc. Natl. Acad. Sci. USA 48: 2039-2043.

[5] Bochner S (1964) Continuous mappings of almost automorphic and almost periodic functions. Proc. Natl. Acad. Sci. USA 52: 907-910.

[6] Blot J , GM Mophoub, GM N'Guérékata and D Pennequina (2009) Weighted pseudo almost automorphic functions and applications to abstract differential equations. Nonlinear Analysis: Theory, Methods and Applications 71 (3/4): 903-909.

[7] Diagania T (2005) Existence of $p$-almost automorphic mild solution to some abstract differential equations. Int. J. Evol. Equ. 1 (1): 57 - 67.

[8] Diagana T (2006) Weighted pseudo almost periodic functions and applications. C. R. Acad. Sci. Paris Ser. I 343 (10): 643646.

[9] Liang J , J Zhang and T-J Xiao (2008) Composition of Pseudo almost automorphic and asymptotically almost automorphic functions. J. Math. Anal. Appl. 340: 1493-1499.

[10] Xiao TJ , J Liang and J Zhang (2008) Pseudo almost automorphic solutions to semilinear differential equations in Banach spaces. Semigroup Forum 76 (3): $518-524$.

[11] N'Guérékata G M (2004) Existence and uniqueness of almost automorphic mild solutions to some semilinear abstract differential equations, Semigroup Forum 69(1): 80-86. 